\section{Symposium IV}

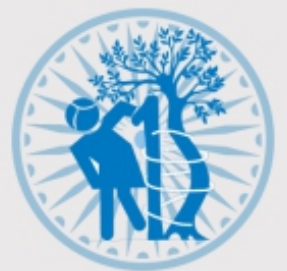

POS I

I JPO

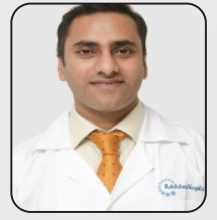

Dr. Vikas Basa

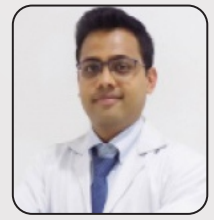

Dr. Avi Shah

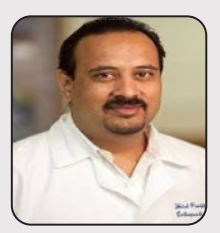

Dr. Shital N Parikh

Address of Correspondence Dr. Shital N Parikh,

Professor of Orthopaedic Surgery, Cincinnati Children's Hospital 3333. Burnet Av Cincinnati, OH. 45229

E-mail: Shital.parikh@cchmc.org

Department of Orthopaedics, Kokilaben Dhirubhai Ambani Hospital and Medical Institute Mumbai, Maharashtra, India.

${ }^{2}$ Department of Orthopaedics, SRCC Childrens Hospital, Mumbai, Maharashtra, India. ${ }^{3}$ Department of Orthopaedic Surgery, Cincinnati Children's Hospital, Burnet Av, Cincinnati, $\mathrm{OH}$.

DOI- 10.13107/ijpo.2021.v07i02.112 | www.ijpoonline.com This is an Open Access article distributed under the terms of the Creative Commons Attribution Non-Commercial-Share Alike 4.0 License(http://creativecommons.org/licenses/ by-nc-sa/4.0) which allows others to remix, tweak, and build upon the work non-commercially as long as appropriate credit is given and the new creation are licensed under the identical terms.

\section{Complications of Lateral Humeral Condyle Fractures in Children}

\author{
Vikas Basa мs ortho, ${ }^{1}$ Avi Shah мs ortho. $^{2}$, Shital N Parikh мD ${ }^{3}$
}

\section{Abstract}

Lateral humeral condyle fractures in children are easily missed and often associated with complications. These complications include malunion, delayed union or nonunion, lateral spur formation, fishtail deformity and growth disturbances. There is also controversy related to best treatment options including closed vs open reduction and K-wire vs screw fixation. Though some complications like malunion are avoidable, others like lateral spur formation are inevitable. Knowledge about these complications would help in counseling patients and their families. The purpose of this article was to review common complications related to these fractures and suggest tips to avoid some of them.

Keywords: Paediatric, Lateral condyle fracture, Internal oblique X-ray, Arthrogram, Anatomical reduction, Complications, Tips to overcome

\section{Introduction}

Lateral Humeral condyle fractures (LHCF) are the second most common elbow fractures in children, after supracondylar humerus fractures. LHCF account for $12 \%-17 \%$ of all distal humerus fractures $[1,2]$. When the fracture is minimally displaced, its diagnosis is challenging and it is missed more often than any other type of elbow fracture in children. In a series of 23 children with LHCF, Flynn et al found that $35 \%$ of nonunions were due to unrecognized fracture at the time of injury [3]. Therefore, a strong clinical suspicion and accurate radiographic interpretation are recommended to diagnose LHCF [4].

The widely used Jakob classification for LHCF is based on the degree of displacement and rotation of the lateral condyle fragment and comprises of three stages [5].

Stage 1: $<2 \mathrm{~mm}$ displacement indicating intact cartilaginous hinge.

Stage 2: 2-4 mm displacement without rotation of the fragment.

Stage 3: $>4 \mathrm{~mm}$ displacement and rotation of the fracture fragment.

The Weiss classification system which is based on degree of displacement and articular congruity can help predict the risk of complications. Compared to surgical treatment outcomes for type 2 fractures (more than $2 \mathrm{~mm}$ displacement but intact articular surface/hinge), type 3 fractures (more than $2 \mathrm{~mm}$ displacement with articular surface breached) had 3 times higher complication rates [6].

LHCF require special attention to ensure appropriate diagnosis and treatment as their clinical and radiographic outcomes are often associated with complications [7]. Understanding these complications would help to minimize some of them as well as help with patient and family counselling. The purpose of this article is to review common complications related to LHCF and suggest tips to recognize them and hopefully avoid them. 


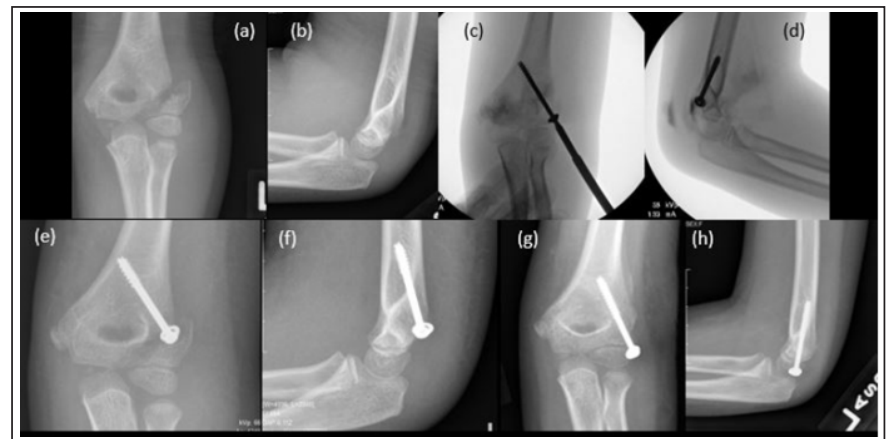

Figure 1: AP ( $1 \mathrm{a})$ and lateral $(1 \mathrm{~b})$ views of a 7.5-year-old girl with a displaced lateral condyle humerus fracture. The AP (1 c) and lateral (1 d) fluoroscopic image shows how the fracture was treated with closed reduction, arthrogram and screw fixation. At one week postoperative follow-up, AP (1 e) and lateral ( $1 \mathrm{f}$ ) radiographs show loss of reduction. The fixation was revised using open reduction and internal fixation $(\mathrm{lg}, \mathrm{h})$.

\section{Loss of Reduction / Non-anatomic Reduction}

Since the lateral condyle of humerus in children is primarily unossified, accurate reduction of LCHF and recognition of inadequate reduction are challenging. The classification and treatment principles are based on amount of fracture displacement on radiographs. As the fracture line is oriented in a posterolateral direction, conventional radiographs frequently underestimate the true displacement. The internal oblique view demonstrates the maximum displacement and should be performed in borderline cases to aid in treatment decision [4]. $4 \mathrm{~mm}$ displacement on the metaphyseal side of the fracture has been used as predictive of articular surface disruption [6]. Though MRI can show the articular surface well, it is seldom required in clinical practice.

At surgery, open reduction can provide direct visualization and confirmation of articular surface reduction. If closed reduction and internal fixation are to be performed, an arthrogram would help to assess the articular surface and aid in internal fixation by delineating the articular surface. A common error during internal fixation is to place the K-wire or screw too proximal and close to the metaphyseal side of fracture since this part is visible on plain radiographs. This can lead to inadequate fixation and subsequent loss of fixation (Figure 1). A properly inserted screw through the cartilaginous fracture fragment may appear to be insufficiently seated, as the screw head would be positioned away from the ossified part of the lateral condyle. Besides suboptimal fixation, other reason for loss of reduction after internal fixation with K-wires may be related to premature removal of K-wires.

\section{Malunion}

Malunion has been amply reported after LCHF in children. Cubitus varus deformity is most common in undisplaced and minimally displaced fractures $[9,10,11]$. One theory is that

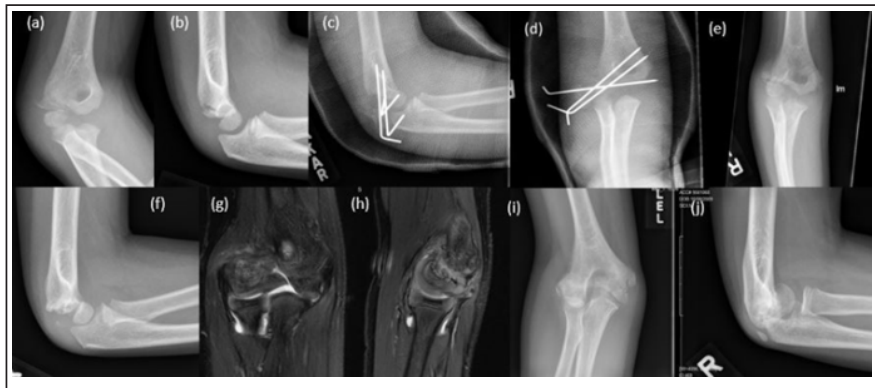

Figure 2: 6-year-old boy with displaced lateral condyle fracture $(2 \mathrm{a}, \mathrm{b})$. The fracture was treated with closed reduction and percutaneous pinning $(2 \mathrm{c}, \mathrm{d})$. At 6 weeks postop, the pins were pulled out. The radiographs show some displacement of the fractured fragment $(2 \mathrm{e}, \mathrm{f})$. MRI shows gap and displacement at the articular surface $(2 \mathrm{~g})$ and flexed position of the lateral condyle fracture fragment $(2 \mathrm{~h})$. Since the patient was asymptomatic, revision surgery was not performed. At 5 years follow-up, AP (2 i) and lateral $(2 \mathrm{j})$ radiographs demonstrate malunited lateral condyle fragment which is flexed, along with osteonecrosis of trochlea. The child is asymptomatic with $15^{\circ}$ loss of extension

these seemingly nondisplaced fractures lose reduction, with the condylar fragment separating distally or tilting medially $[11,12]$. However, Tan et al attributed this deformity to prolonged hyperaemia and growth stimulation of lateral condyle [13]. Cubitus valgus, which is much less common, is believed to be caused by lateral physeal arrest, non-union, avascular necrosis or malunion $[12,13]$. Skak et al reported that none of their 7 patients with varus deformity were more than 8 years age at time of injury, whereas valgus position in 8 patients was found with equal frequency among all age groups [8].

Closed reduction and percutaneous internal fixation may lead to malunion if there is suboptimal visualization of fracture reduction on fluoroscopy (Figure 2). When in doubt, an arthrogram or open reduction approach can prevent malunion by direct visualization of the articular surface reduction.

Minor deformities and those not limiting the elbow function can be treated conservatively [14]. Supracondylar humeral osteotomy has been used to improve symptomatic cubitus varus/valgus alignment in pediatric elbow malunions. The advantage of such an osteotomy is the minimal risk to the articular cartilage, distal humerus blood supply and elbow motion. Although proximal osteotomy can improve varus and valgus malalignment, it does not address elbow motion deficits or prevent long-term arthrosis from articular malunion.

For LCHF malunion involving the articular surface of the distal humerus, Bauer et al [15] reported on intra-articular corrective osteotomy to improve elbow motion, pain and radiographic alignment. They emphasized careful selection of patients and meticulous surgical technique. The authors observed significant improvement in the elbow range of motion and suggested that intra-articular corrective osteotomy can be 


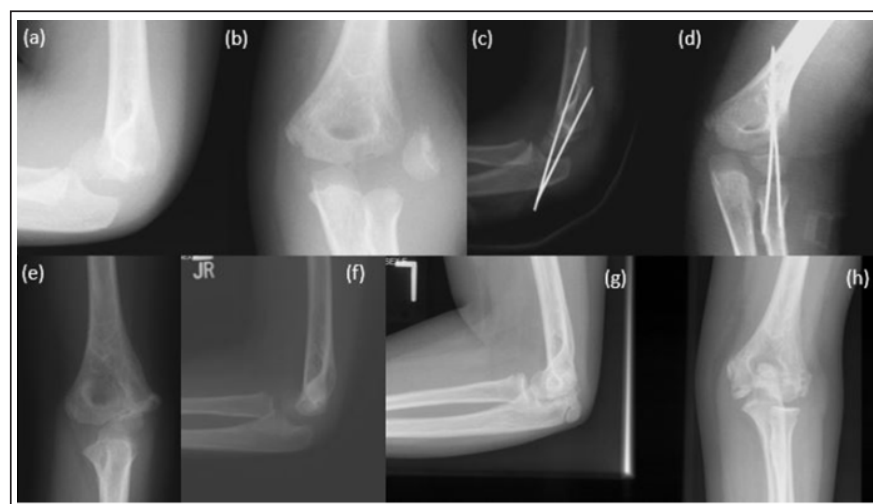

Figure 3: A 7-year-old girl with completely displaced lateral condyle fracture $(3 \mathrm{a}, \mathrm{b})$. The fracture was treated with open reduction and pinning $(3 \mathrm{c}, \mathrm{d})$. Radiographs at 5 months postop, show acceptable fracture alignment $(3 \mathrm{e}, \mathrm{f})$. At 3 years follow-up, the fracture is united but there is mild increase in the valgus alignment at the elbow due to lateral-sided growth arrest $(3 \mathrm{~g}, \mathrm{~h})$. CT scan is in the next figure. Image courtesy of Charles T. Mehlman, DO, Cincinnati Children's Hospital.

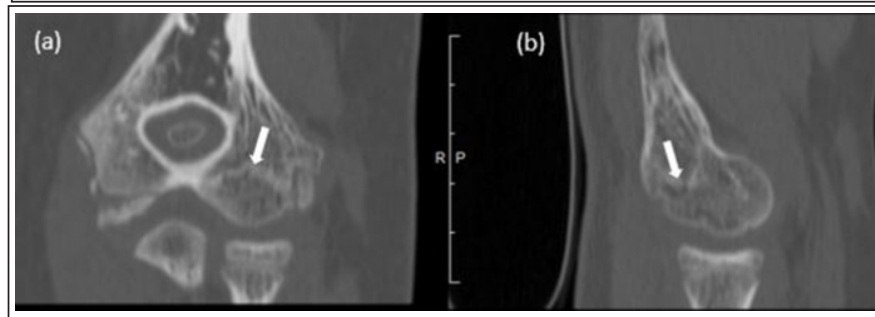

Figure 4: CT scan of the same patient as in Figure 3 shows growth arrest (white arrows) on coronal and sagittal section. Also seen is some avascular necrosis of trochlea. Image courtesy of Charles T. Mehlman, DO, Cincinnati Children's Hospital.

successful when performed in the first year after the initial fracture. They cautioned performing this procedure in Milch type 2 fracture malunion as there was less satisfactory improvement in elbow motion and alignment.

\section{Growth Arrest}

Growth arrest or premature epiphyseal closure after LCHF are uncommon and may not be obvious for years $[10,12,17,18]$. Tan et al reported a growth arrest rate of 5.4\%. As LHCF are typically Salter-Harris type IV fractures [12, 13] with the fracture line extending through the metaphysis, physis, and epiphysis, growth arrest can occur if there is initial or persistent displacement after treatment. A number of factors may contribute to premature physeal arrest, including fracture displacement, soft tissue injury and vascular insult, nonanatomic reduction, and compression across the physis with implants [19]. Since the distal humerus has limited growth (compared to the proximal humerus), the sequelae of growth arrest may be minimal or may not manifest until later. Mehlman et al [19] reported 3 cases of physeal arrest after a mean of 2.6 years after injury (Figure 3, 4). All patients had increased carrying angle; 2 were asymptomatic and 1 patients had pain and loss of terminal extension. The authors recommend diligent monitoring of patients and long-term follow-up till skeletal maturity. If this is not feasible, the family should be counselled about the possibility of late deformities and to follow-up in case such deformities occur.

\section{Loss of Motion}

Loss of terminal range of motion is more prevalent with LCHF fractures than with other elbow fractures. Minor $\left(5^{\circ}\right)$ loss of flexion-extension motion has been reported in up to $40 \%$ patients after LCHF and $15.6 \%$ patients had $>15^{\circ}$ loss of flexion-extension movement in one series [20]. Tan et al [13] reported that extension and flexion limitations affected 9.7\% and $11.5 \%$ of LCHF respectively. Compared to supracondylar humerus fractures which start healing at 3 weeks post-injury, LCHF may take about 4 to 6 weeks for initial healing to be apparent. It is not infrequent to have long-arm cast immobilization for about 6 weeks following LCHF treatment. Besides duration of immobilization, other reported risk factors associated with loss of motion include intra-articular fracture and scar tissue, inappropriate treatment, K-wires as opposed to screw fixation and delayed presentation [13]. Though most motion deficits decrease at follow-up, Sinikumpu et al [20] reported $>10^{\circ}$ loss of elbow motion in 9 of 32 patients and $>10^{\circ}$ loss of forearm rotation in 6 of 32 patients at a mean follow-up of 12 years. Anatomic reduction of the fracture and screw fixation could allow for early mobilization of elbow thereby minimizing loss of motion in patients with LCHF. After initial fracture healing, our preference is to allow the patient to regain range of motion without formal physical therapy. Those with significant loss of motion may benefit from formal physical therapy after $2-3$ months. Very rarely, surgery may be required for treatment of elbow arthrofibrosis $[21,22]$.

\section{Delayed Union}

Delayed union is defined as a lateral condyle fracture that does not exhibit radiological evidence of fracture healing by eighth week of follow up [23] (Figure 5). Risks for delayed healing include multiple attempts at reduction and amount of residual displacement after reduction [23]. Tan et al. [13] reported delayed union in patients treated conservatively or those that were inadequately reduced when managed operatively. They recommend prolonged immobilisation or open reduction and internal fixation with or without bone grafting.

\section{Lateral Spur / Overgrowth}

The most frequently seen complication after LCHF treatment is the lateral spur or overgrowth $[7,13,24,25]$ (Figure 6). This complication is frequently seen on radiographs and sometimes on clinical evaluation. When there is focal overgrowth, it 


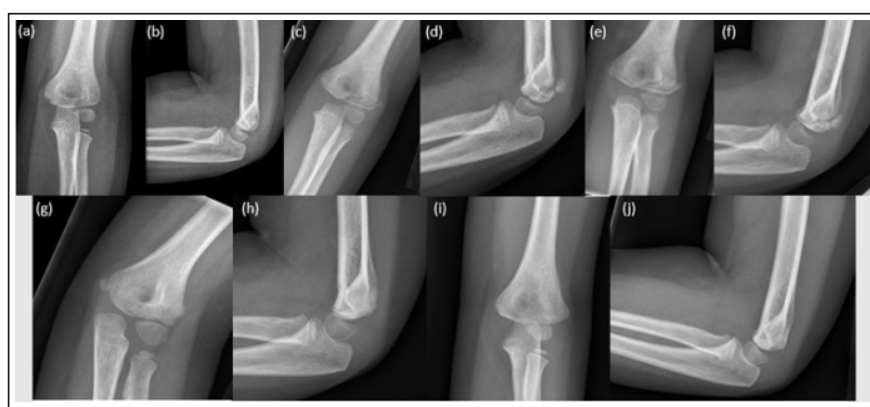

Figure 5: A 4-year-old girl child with an undisplaced lateral condyle fracture $(5 \mathrm{a}, \mathrm{b})$. At 5 weeks, the fracture appears to be displaced more than before $(5 \mathrm{c}, \mathrm{d})$, without any signs of healing. At 8 weeks $(5 \mathrm{e}, \mathrm{f})$, there is some callus formation but fracture line is distinctly visible. For a young child, this would suggest delayed union. At 12 weeks, there is complete healing of the fracture $(5 \mathrm{~g}, \mathrm{~h})$. At 10 months post-injury, there is remodelling of the fracture $(5 \mathrm{I}, \mathrm{j})$.

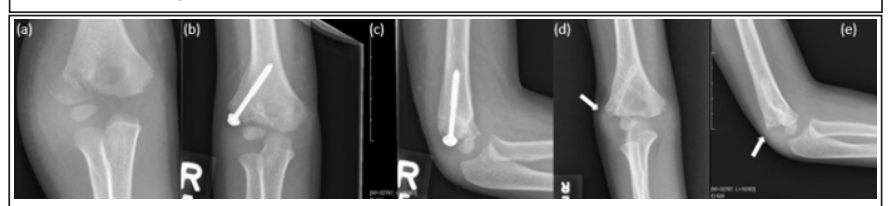

Figure 6: 5-year-old boy with displaced fracture of the lateral condyle $(6 \mathrm{a})$. It was treated with open reduction and internal fixation $(6 \mathrm{~b}, \mathrm{c})$. After removal of hardware (6 d, e), the posterolateral prominence or spur (white arrow) is visible on the radiographs, despite anatomic reduction and healing of the fracture. The posterolateral spur is common after lateral condyle fracture treatment.

presents as a bump whereas generalized overgrowth may give rise to pseudo-cubitus varus [8]. Pribaz et al reported that $73 \%$ of patients in their series of 212 patients had evidence of lateral spurring after LCHF treatment. The presence and size of the spur correlated with initial fracture displacement but did not correlate with age. The mean initial displacement of fracture was $3.3 \mathrm{~mm}$ in those who developed a spur as compared to 1.1 $\mathrm{mm}$ in those did not develop a spur. Mild spurring (0-10\% increase in width of distal humerus) was observed in $43 \%$, moderate (10-20\% increase in width) in 38\% and severe (>20\% increase in width) in $19 \%$ of patients. $59 \%$ patients with lateral spur were treated conservative and $41 \%$ patients had surgery. There was no difference in spur formation between patients who underwent closed reduction and K-wire fixation as compared to those who underwent open reduction and K-wire fixation. Some studies have reported decreased rate of lateral spur formation with cannulated screw fixation $[24,27,28,25]$. Though the exact aetiology is unknown, lateral spurring may be related to inadequate reduction, local hyperaemia and bony overgrowth from lateral condylar stimulation or from displaced periosteum and damaged soft tissues $[7,13,15,25]$. The lateral spur has not shown any correlation with pain, loss of motion or functional consequences. Hence no treatment is generally required. The long-term natural history of lateral spur is not known but based on the growth pattern of the distal humerus, it is unlikely to remodel and more likely to persist at skeletal maturity $[7,24,26]$. It is advisable to inform the patients and families about the likelihood of lateral spur formation prior to initiation of treatment.

\section{Fish Tail deformity/AVN}

Avascualar necrosis is a rare and devastating complication and poses a vexing problem to treating surgeon. Minor degrees of AVN and slight deepening of trochlear groove, however, is more commonly reported in recent literature [8]. When significant, it leads to a concavity which is visible on AP radiographs where the lateral trochlear ossification centre fails to develop because of disrupted blood supply to the trochlea, leading to fish tail deformity $[7,13,24,29,30]$. It occurs when the lateral vascular tributaries are disrupted, whereas frank osteonecrosis occurs when both the medial and lateral blood supplies are injured. Tan et al [13] reported $14 \%$ incidence of fishtail deformity and $1 \%$ incidence of AVN in their systematic review. Though it is recommended to avoid posterior soft tissue stripping when trying to visualise the fracture to decrease vascular insult, AVN has been reported after nonsurgical treatment as well $[7,24,30]$.

Increased risk of AVN has been correlated with displaced Milch Type 2 fractures, Jakob Type 3 fractures as well as delayed treatment for these fractures [13]. Instability, mal-reduction and lack of compression at fracture site have all been reported to increase the risk of AVN $[6,8,9,13,31]$.

The manifestation of AVN and deformity occurs late; about 4-8 years after the initial injury and can be difficult to evaluate early. It can lead to limited ROM, pain, loose bodies, and/or cubitus valgus deformity, leading to degenerative arthritis, and ulnar neuropathy $[7,8,13,24,30,32]$. MRI can help to visualize AVN before radiographs in patients with late developing stiffness or pain $[7,24,30]$. Treatment for this rare complication can be challenging. The spectrum of treatment includes observation for asymptomatic patients, removal of loose bodies, epiphysiodesis, osteotomy, and/or ulnar nerve transposition $[29,30]$.

Tonolino et al described osteonecrosis of two types, type A with lesions lateral to medial crista of trochlea which are more likely to lose range of motion and develop arthritis. They typically do not develop angular deformities. Type B lesions which involve the entire trochlea and part of the metaphysis are more likely to create a progressive varus deformity [30]. Glotzbecker et al [30] reported on 15 cases of fishtail deformities; 4 were related to LCHF at a follow-up of 3.9 years. Their report lacks details and specific recommendations for LCHF. A treatment algorithm was suggested in which children with functional ROM (25-130), minimal symptoms and small 
defects were treated with observation. Children with severe symptoms and deformity were treated surgically by joint debridement and growth modulation/osteotomy for deformity correction. They reported good short term results but at long term follow up, $43 \%$ had persistent pain and only $14 \%$ regained full ROM.

\section{Screws vs Pins}

Good results have been reported with both K-wires and screw fixation for LCHF in the literature $[7,13,24,33,34,35,36]$. Recent studies have favoured screw fixation over K-wires due to reduced cast time, earlier mobilisation, reduced infection rates, fewer non-union rates, faster time to union due to compression at fracture site and overall better motion at follow up $[7,13,24$, $27,33,34]$. The disadvantage of screw fixation is that it requires a second surgery for removal and may increase overall treatment costs. The disadvantage of $\mathrm{K}$-wires is the higher risk of pin tract infections and earlier removal which may lead to non-union, delayed union or malunion.

The other controversy is whether to bury the K-wires or to leave them out of the skin. Qin et al, in their meta-analysis, found no significant differences related to infection, delayed union reoperation or total complications in buried vs unburied $\mathrm{K}$-wires. Unburied K-wires were not associated with higher infection rates and had the benefit of early removal and cost savings $[37,38,39]$.

\section{Rare Complications}

Tan et al [13] in their systematic review reported $10 \%$ incidence of neurological deficits with the commonest being tardy ulnar nerve palsy associated with non-union and cubitus valgus deformity. Neuropraxia of the anterior and posterior interosseous nerves have been reported as rare occurrences $[6$, $7,13,31,32,40]$. Other complications reported in literature with LCHF are persistence of pain $[6,7,13,24,31,32,40]$, radio-capitellar osteoarthritis 3,13 and heterotopic ossification13,41.

\section{Conclusion}

The propensity for lateral condyle fractures to develop complications is well established. Missed fractures and inadequate management are the most common causes of nonunion and deformity; thus, a high index of suspicion and adequate clinical evaluation with appropriate radiographic views are necessary. An arthrogram can aid in confirming adequate closed reduction. Understanding the common pitfalls and complications can help minimize them and allow surgeons to counsel families appropriately.

Declaration of patient consent: The authors certify that they have obtained all appropriate patient consent forms. In the form, the patient has given his consent for his images and other clinical information to be reported in the Journal. The patient understands that his name and initials will not be published, and due efforts will be made to conceal his identity, but anonymity cannot be guaranteed.

Conflict of interest: Nil; Source of support: None

\section{References}

1. Bhandari M, Tornetta $\mathrm{P}$ and Swiontkowksi MF The Evidence-Based Orthopaedic Trauma Working Group. Displaced lateral condyle fractures of the distal humerus.J Orthop Trauma 2003; 17: 306-308.

2. Hardacre JA, Nahigian SH, Froimson AI and Brown JE. Fractures of the lateral condyle of the humerus in children.J Bone Joint Surg (Am) 1971; 53: $1083-1095$.

3.Flynn JC: Nonunion of slightly displaced fractures of the lateral humeral condyle in children: An update.J Pediatr Orthop 1989;9(6):691-696.

4. Song KS, Kang $\mathrm{CH}$, Min BW, Bae $\mathrm{KC}$ and $\mathrm{Cho} \mathrm{CH}$. Internal oblique radiographs for diagnosis of nondisplaced or minimally displaced lateral condylar fractures of the humerus in children.J Bone Joint Surg Am 2007; 89: $58-63$.

5. Jakob R, Fowles JV, Rang M and Kassab MT. Observations concerning fractures of the lateral humeral condyle in children. J Bone Joint Surg $(\mathrm{Br})$ $1975 ; 57: 430-436$.

6. Weiss JM, Graves S, Yang S, et al. A new classification system predictive of complications in surgically treated pediatric humeral lateral condyle fractures.J Pediatr Orthop. 2009;29: 602-605.
7. Schroeder K, Gilbert S, Ellington M, Souder C, Yang S. Pediatric Lateral Humeral Condyle Fractures.JPOSNA. 2020 May 3;2(1).

8. Skak SV, Olsen SD, Smaabrekke A: Deformity after fracture of the lateral humeral condyle in children.J Pediatr Orthop B 2001; 10:142-152.

9. Rutherford A: Fractures of the lateral humeral condyle in children. J Bone Joint Surg (Am) 1985;67(6):851-856.

10. Badelon O, Bensahel H, Mazda K, Vie P: Lateral humeral condylar fractures in children: A report of 47 cases. J Pediatr Orthop 1988;8(1):31-34.

11. So YC, Fang D, Leong JC, Bong SC: Varus deformity following lateral humeral condylar fractures in children.J Pediatr Orthop 1985;5(5):569-572.

12. Tejwani N, Phillips D, Goldstein RY. Management of lateral humeral condylar fracture in children.J Am Acad Orthop Surg. 2011; 19:350-358.

13. Tan SH, Dartnell J, Lim AK, Hui JH. Paediatric lateral condyle fractures: a systematic review. Arch Ortho Trauma Surgery. 2018 Jun 1;138(6):809-17.

14. Wirmer J, Kruppa C, Fitze G. Operative treatment of lateral humeral condyle fractures in children. EurJ Pediatr Surg 2012:22(4):289-294. 
15. Bauer AS, Bae DS, Brustowicz KA, Waters PM. Intra-articular corrective osteotomy of humeral lateral condyle malunions in children: early clinical and radiographic results.J Pediatr Orthop. 2013 Jan 1;33(1):20-5.

16. Dhillon KS, Sengupta S, Singh BJ. Delayed management of fracture of the lateral humeral condyle in children. Acta Orthop Scand. 1988; 59:419-424.

17. Beaty JH, Kasser JR. Rockwood \& Wilkens fractures in children. 7th ed. Philadelphia. Wolters Kluwer/Lippincott Williams and Wilkens; 2010.

18. Wadsworth TG. Injuries of the capitular (lateral humeral condylar) epiphysis. Clin Orthop Relat Res. 1972; 85:127-142.

19. Cates RA, Mehlman CT. Growth arrest of the capitellar physis after displaced lateral condyle fractures in children. J Pediatr Orthop. 2012 Dec 1;32(8): e57-62.

20. Sinikumpu, J-J, Pokka, T, Victorzon, S, et al.: Paediatric lateral humeral condylar fracture outcomes at twelve years' follow-up as compared with age and sex matched paired controls. Int Orthop 2017; 41:1453-1461.

21. Papandrea R, Waters PM. Posttraumatic reconstruction of the elbow in the pediatric patient. Clin Orthop Relat Res. 2000; 370:115-26.

22. Mader K, Koslowsky TC, Gausepohl T, Pennig D. Mechanical distraction for the treatment of posttraumatic stiffness of the elbow in children and adolescents: surgical technique. J Bone Joint Surg. 2007 Mar 1;89(2_suppl_1):26-35.

23. Salgueiro L, Roocroft JH, Bastrom TP, Edmonds EW, Pennock AT, Upasani VV, Yaszay B. Rate and risk factors for delayed healing following surgical treatment of lateral condyle humerus fractures in children. J Pediatr Orthop. 2017 Jan 1;37(1):1-6.

24. Abzug JM, Dua K, Kozin SH, Herman MJ. Current concepts in the treatment of lateral condyle fractures in children. J Am Acad Orthop Surg. 2020 Jan 1;28(1): e9-19.

25. Pribaz JR, Bernthal NM, Wong TC, Silva M: Lateral spurring (overgrowth) after pediatric lateral condyle fractures.J Pediatr Orthop 2012; $32: 456-460$.

26. Leonidou A, Chettiar K, Graham S, et al: Open reduction internal fixation of lateral humeral condyle fractures in children: A series of 105 fractures from a single institution. Strateg Trauma Lim Reconstr. 2014; 9:73-78.

27. Shirley E, Anderson M, Neal K, Mazur J: Screw fixation of lateral condyle fractures: Results of treatment.J Pediatr Orthop 2015; 35:821-824.

28. Li WC, Xu RJ: Comparison of Kirschner wires and AO cannulated screw internal fixation for displaced lateral humeral condyle fracture in children. Int Orthop 2012; 36:1261-1266.
29. Narayanan S, Shailam R, Grottkau BE, Nimkin K: Fishtail deformity: A delayed complication of distal humeral fractures in children. Pediatr Radiol 2015;45: 814-819.

30. Glotzbecker MP, Bae DS, Links AC, Waters PM. Fishtail deformity of the distal humerus: a report of 15 cases.J Pediatr Orthop. 2013 Sep 1;33(6):5927.

31. Hasler CC, von Laer L Prevention of growth disturbances after fractures of the lateral humeral condyle in children. J Pediatr Orthop B 2001: 10(2):123-130.

32. Foster DE, Sullivan JA, Gross RH Lateral humeral condylar fractures in children.J Pediatr Orthop 1985: 5(1):16-22.

33. Gilbert SR, MacLennan PA, Schlitz RS, Estes AR. Screw versus pin fixation with open reduction of pediatric lateral condyle fractures. J Pediatr Orthop B.2016 Mar 1;25(2):148-52.

34. Stein BE, Ramji AF, Hassanzadeh H, Wohlgemut JM, Ain MC, Sponseller PD. Cannulated lag screw fixation of displaced lateral humeral condyle fractures is associated with lower rates of open reduction and infection than pin fixation.J Pediatr Orthop. 2017 Jan 1;37(1):7-13.

35. Birkett N, Al-Tawil K, Montgomery A. Functional Outcomes Following Surgical Fixation of Paediatric Lateral Condyle Fractures of the Elbow - A Systematic Review. Orthop Res Rev. 2020 Mar 6; 12:45-52.

36. Wendling-Keim DS, Teschemacher S, Dietz HG, Lehner M. Lateral Condyle Fracture of the Humerus in Children: Kirschner Wire or Screw Fixation? EurJ Pediatr Surg. 2020 Jul 28.

37. Qin YF, Li ZJ, Li CK, Bai SC, Li H. Unburied versus buried wires for fixation of pediatric lateral condyle distal humeral fractures: A meta-analysis. Medicine.2017 Aug;96(34).

38. Ormsby NM, Walton RD, Robinson S, Brookes-Fazakerly S, Chang FY, McGonagle L, Wright D. Buried versus unburied Kirschner wires in the management of paediatric lateral condyle elbow fractures: a comparative study from a tertiary centre.J Pediatr Orthop B. 2016 Jan 1;25(1):69-73.

39. De SD, Bae DS, Waters PM. Displaced humeral lateral condyle fractures in children: should we bury the pins? J Pediatr Orthop. 2012 Sep $1 ; 32(6): 573-8$

40. Thomas DP, Howard AW, Cole WG, Hedden DM. Three weeks of Kirschner wire fixation for displaced lateral condylar fractures of the humerus in children.J Pediatr Orthop 2001:21(5):565-569.

41. Andrey V, Tercier S, Vauclair F, Bregou-Bourgeois A, Lutz N, Zambelli PY. Lateral condyle fracture of the humerus in children treated with bioabsorbable materials. Sci World J 2013: 9:869418.

How to Cite this Article

Basa V, Shah A, Parikh SN | Complications of Lateral Humeral Condyle Fractures in Children

| International Journal of Paediatric Orthopaedics | May-August 2021; 7(2): 42-47. 